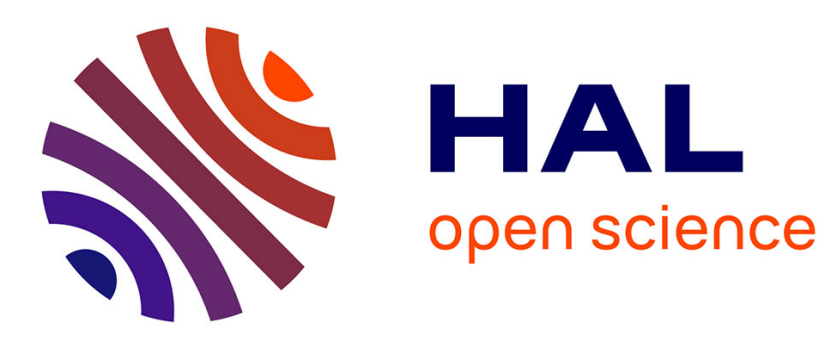

\title{
Alignment and distribution is NOT (always) NP-hard.
}

Vincent Boudet, Fabrice Rastello, Yves Robert

\section{To cite this version:}

Vincent Boudet, Fabrice Rastello, Yves Robert. Alignment and distribution is NOT (always) NPhard.. [Research Report] LIP RR-1998-30, Laboratoire de l'informatique du parallélisme. 1998, 2+18p. hal-02101994

\section{HAL Id: hal-02101994 \\ https://hal-lara.archives-ouvertes.fr/hal-02101994}

Submitted on 17 Apr 2019

HAL is a multi-disciplinary open access archive for the deposit and dissemination of scientific research documents, whether they are published or not. The documents may come from teaching and research institutions in France or abroad, or from public or private research centers.
L'archive ouverte pluridisciplinaire HAL, est destinée au dépôt et à la diffusion de documents scientifiques de niveau recherche, publiés ou non, émanant des établissements d'enseignement et de recherche français ou étrangers, des laboratoires publics ou privés. 


\section{Alignment and distribution is NOT (always) NP-hard}

Vincent BOUDET

Fabrice RASTELLO

Juillet 1998

Yves ROBERT 


\title{
Alignment and distribution is NOT (always) NP-hard
}

\author{
Vincent BOUDET \\ Fabrice RASTELLO \\ Yves ROBERT
}

Juillet 1998

\begin{abstract}
In this paper, an efficient algorithm to simultaneously implement array alignment and data/computation distribution is introduced and evaluated. We re-visit previous work of $\mathrm{Li}$ and Chen $[13,14]$, and we show that their alignment step should not be conducted without preserving the potential parallelism. In other words, the optimal alignment may well sequentialize computations, whatever the distribution afterwards. We provide an efficient algorithm that handles alignment and data/computation distribution simultaneously. The good news is that several important instances of the whole alignment/distribution problem have polynomial complexity, while alignment itself is NP-complete [13].
\end{abstract}

Keywords: compilation techniques, parallel loops, alignment, distribution, "the owner computes" rule.

\section{Résumé}

Dans ce rapport, un algorithme efficace est présenté et évalué pour résoudre simultanément l'alignement des tableaux et la distribution des données et des calculs. Nous revisitons les travaux précédents de Li et Chen [13, 14], et nous montrons que leur recherche d'un alignement ne doit pas être conduite sans préserver le parallélisme potentiel. En d'autres termes, l'alignement optimal peut séquentialiser les calculs quelle que soit la distribution choisie ensuite. Nous présentons un algorithme efficace qui tient compte simultanément de l'alignement et de la distribution des données et des calculs. La bonne nouvelle est que plusieurs instances du problème d'alignement/distribution ont une complexité polynomiale alors que l'alignement lui-même est NP-complet [13].

Mots-clés: techniques de compilation, boucles parallèles, alignement, distrib ution, règle du "the owner computes". 


\title{
Alignment and distribution is NOT (always) NP-hard*
}

\author{
Vincent Boudet, Fabrice Rastello and Yves Robert \\ LIP, URA CNRS 1398 \\ Ecole Normale Supérieure de Lyon, 69364 Lyon Cedex 07, France \\ e-mail: [Vincent.Boudet, Fabrice.Rastello, Yves.Robert] dens-lyon.fr
}

\begin{abstract}
In this paper, an efficient algorithm to simultaneously implement array alignment and data/computation distribution is introduced and evaluated. We re-visit previous work of Li and Chen [13,14], and we show that their alignment step should not be conducted without preserving the potential parallelism. In other words, the optimal alignment may well sequentialize computations, whatever the distribution afterwards. We provide an efficient algorithm that handles alignment and data/computation distribution simultaneously. The good news is that several important instances of the whole alignment/distribution problem have polynomial complexity, while alignment itself is NP-complete [13].
\end{abstract}

Key words: compilation techniques, parallel loops, alignment, distribution, "the owner computes" rule.

Corresponding author: Yves Robert

LIP, Ecole Normale Supérieure de Lyon, 69364 Lyon Cedex 07, France

Phone: + 33472728037 , Fax: + 33472728080

E-mail: Yves.Robert@ens-lyon.fr

*This work was supported by the CNRS-ENS Lyon-INRIA project ReMaP and by the Eureka Project EuroTOPS. 


\section{Introduction}

Compile-time techniques for mapping arrays and computations onto distributed memory machines have focused a large research effort recently, as illustrated by the survey paper of Ayguadé, Garcia and Kremer [5]. Several methods and tools have been presented since the reference paper of Li and Chen $[13,14]$, who have studied the problem of aligning arrays so as to minimize communications. Because Li and Chen have shown the alignment problem to be NP-complete (in the number of data arrays and statements within the loop nest), heuristics or costly (exponential) algorithms, such as Integer Linear Programming, have been introduced. We briefly survey the related literature in Section 2.

In this paper we re-visit previous work of $\mathrm{Li}$ and Chen $[13,14]$, and we show that their alignment step should not be conducted without preserving the potential parallelism. In other words, the optimal alignment may well sequentialize computations, whatever the distribution afterwards. We provide an efficient algorithm that handles alignment and data/computation distribution simultaneously. The good news is that several important instances of the whole alignment-distribution problem have polynomial complexity, while alignment itself is NP-complete [13].

We take as input a loop nest, possibly non perfect, where parallelism has been made explicit, e.g. after applying the Allen and Kennedy parallelization algorithm [2]. We construct a new graph, the alignment-distribution graph, which replaces $\mathrm{Li}$ and Chen's component affinity graph. Using this graph, we are able to determine which parallel loop(s) and which array dimension(s) should be distributed to the processors so as to preserve parallelism while minimizing communications. Our alignment-distribution graph is weighted, and the weights represent estimates of the communication costs: it is a very flexible approach, and we are able to take advantage of recent results on modeling such communication costs accurately [7, 4, 3, 11]. Because the choice of the distributed loops provides kind of a "reference" pattern, the alignment step is conducted according to this choice, and the complexity to finding the optimal solution reduces to a fast (polynomial) path algorithm on the alignment-distribution graph. This is a very nice result for the practical applicability of our approach (again, previous techniques aimed at solving a NP-complete problem).

The paper is organized as follows: we start with a motivating example in Section 2. We use the example to summarize the approach of $\mathrm{Li}$ and Chen $[13,14]$ and to point out its limitations. We briefly review the existing literature in Section 3. We describe our new algorithm, and we state complexity results, in Section 4. We give some final remarks in Section 5.

\section{Motivation}

We use a simple example to explain why aligning arrays and distributing parallel loops should be dealt with simultaneously.

\section{Example 1}

$$
\begin{aligned}
& \text { for } i=2 \text { to } n \text { do } \\
& \text { for } / / j=i+1 \text { to } n \text { do } \\
& \qquad \begin{array}{l}
S_{1}: a(i, j)=b(i, j)+a(i-1, j) \\
S_{2}: b(j, i)=a(j, j)+1
\end{array} \\
& \text { end for } / /
\end{aligned}
$$


To check that the second loop on $j$ is indeed parallel, we can use a dependence analysis tool like Tiny [18]. Using such a tool, we check that there is only one flow dependence of level 1 from $S_{1}$ to itself, which is due to $a$. The reduced dependence graph for Example 1 is depicted in Figure 1.

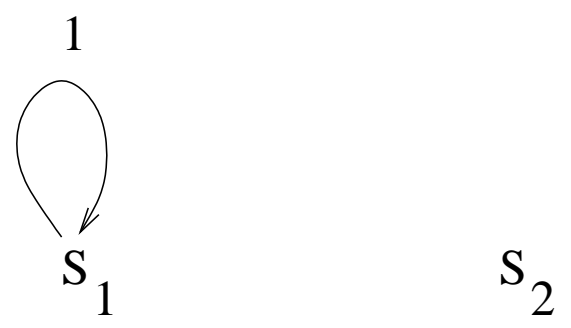

Figure 1: The reduced dependence graph (using dependence levels) for Example 1.

First we review Li and Chen's approach $[13,14]$ through Example 1 . Then we explain why their technique may kill the potential parallelism.

\subsection{Li and Chen's component affinity graph}

We represent in Figure 2 the component affinity graph (CAG) that $\mathrm{Li}$ and Chen [14, 13] would derive for Example 1. We informally explain how the CAG is built using the example. The CAG contains 2 columns of 2 nodes, because they are 2 arrays $a$ and $b$ (hence 2 columns) of dimension 2 each (hence two nodes in each column). Node $a 1$ represents the first dimension of array $a$, and so on. There is an edge between two nodes, i.e. between two dimensions of different arrays, if, roughly speaking, the subscripts of these dimensions are the same up to a translation by a constant, and if these arrays appear on both sides of the same assignment. The CAG is undirected. Self references are not taken into account. In our example, there is an edge between nodes $a 1$ and $b 1$ because of statement $S_{1}$ : the same subscript $i$ appears in the first dimension of $a$ and $b$. In general, when the same subscript, up to a translation by a constant, appears in dimension $i_{x}$ of array $x$ and in dimension $i_{y}$ of array $y$, these two dimensions are said to have an affinity relationship, and we draw an edge between the corresponding nodes. Similarly, due to $S_{1}$ again, there is an edge between $b 2$ and $a 2$. Because self references are not taken into account, the occurrence of $a(i-1, j)$ in the right hand side has no impact on the graph. The intuitive idea is that edges imply an alignment preference between the corresponding arrays. The term alignment may well be understood here as an HPF ALIGN directive [10] onto a virtual template. Aligning arrays according to the edges will reduce, or even suppress (as in statement $S_{1}$ ), the possible communications induced by the distribution of the arrays onto parallel processors.

Statement $S_{2}$ introduces some complication, because the same index $j$ appears in the first dimension of $a$ on the left hand side, and in both dimensions of $b$ on the right hand side. The two edges $(a 1, b 1)$ and $(a 1, b 2)$ are said to be competing.

The CAG is weighted: edges are valued according to the strength of preference. A competing edge has weight $\varepsilon$, a value much smaller than 1 . The weight of an edge between nodes indexed by a spatial variable (a subscript of a parallel loop, like $j$ in Example 1 ) is 1 . Finally, the weight of an edge between nodes indexed by a temporal variable (a subscript of a sequential loop, like $i$ in Example 1) is $\infty$. We are led to the graph of Figure 2. If there are several edges between two nodes, we only keep one, whose weight is the sum of all edge weights between the two nodes.

$\mathrm{Li}$ and Chen $[14,13]$ state the alignment problem as follows: partition the nodes of all columns into disjoint subsets that represent aligned dimensions. The rule of the game is that no two nodes 


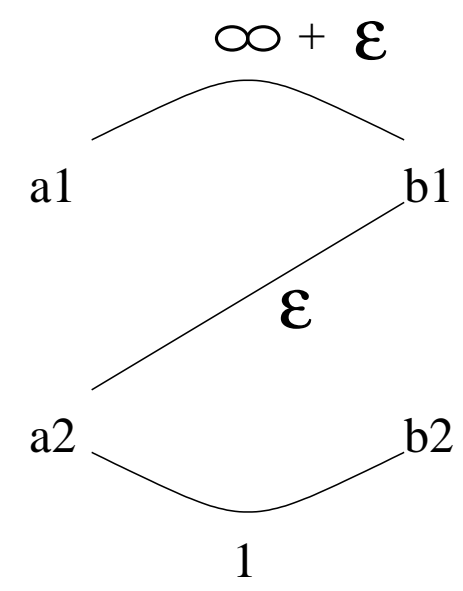

Figure 2: The component affinity graph for Example 1.

of the same column are in the same subset. The objective is to minimize the sum of the edge weights between subsets. Unfortunately, the problem is NP-complete in the size of the CAG (Li and Chen use a reduction from MAX-CUT [6]). To compute a satisfactory alignment, Li and Chen use a greedy heuristic based upon bipartite matching [14]. For Example 1, their heuristic leads to the optimal (minimal-weight) solution, namely aligning $a 1$ with $b 1$ and $a 2$ with $b 2$. In other words arrays $a$ and $b$ are directly superimposed onto the same template.

\subsection{Distributing parallel loops}

The previous alignment, however, causes all the potential parallelism to be lost when it comes to distributing array elements onto processors! To see why, consider the following two possible data distributions:

Distributing the first dimension This means that rows of arrays $a$ and $b$ are distributed to processors: elements $a(i, j)$ and $b(i, j)$, for $1 \leq j \leq n$, are stored in (virtual) processor $P_{i}$. This causes statement $S_{1}$ to be executed sequentially: given a value of the first loop index $i$, all iterations of the second loop index $j$ are computed by the same processor $P_{i}$.

Distributing the second dimension Quite similarly, distributing columns of $a$ and $b$ to processors will lead statement $S_{2}$ to be executed sequentially.

To summarize, the best alignment, as computed by Li and Chen, turns out to kill the parallelism. We claim that the alignment step should be conducted while having parallelism in mind: distributing parallel loops to processors is the true priority. A good alignment can reduce or suppress communications, but what if it leads to gather all parallel computations onto the same processor, as in our example?

We informally explain our approach using Example 1. See Section 4 for a complete description of our algorithm. Assume we target a one-dimensional processor grid. The highest priority is to distribute parallel computations, i.e. instances of the parallel loop $j$, on processors. In the example there is not much freedom: we distribute columns of $a$ and rows of $b$ to processors: processor $P_{j}$ receives $a(i, j)$ and $b(j, i)$ for all $1 \leq i \leq n$. Owing to this distribution, for each instance of the external loop $i$, we distribute the parallel computations of loop $j$ to processors. There remains some communications: for each instance $i$ of the external loop, because of statement $S_{1}$, the $i$-th 
row of $b$ must be scattered from processor $P_{i}$ to all processors. But parallelism has been preserved. Our approach does lead to this solution, based upon an alignment-distribution graph that privileges parallel loops. The alignment-distribution graph for Example 1 is represented in Figure 3 . It is built as follows: there are 4 array dimension nodes, one per array and per dimension, as in Li and Chen's CAG, plus an additional loop node for the parallel $j$ loop. There is an edge between the loop node and an array dimension node if distributing both of them onto the processors induces communications. Edge weigh correspond to (estimated) communication costs. In Figure 3, $G a$ stands for Gather, and Sc for Scatter.

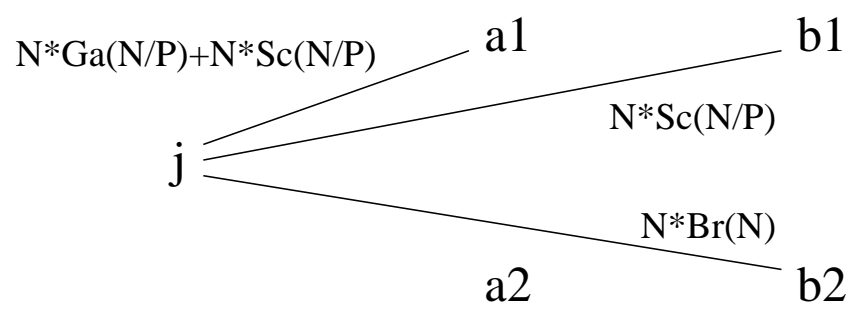

Figure 3: The alignment-distribution graph for Example 1.

The detailed construction of the graph as well as our solution to the problem are described in Section 4. We conclude our study of Example 1 with a few important remarks:

Remark 1: "the owner-computes" rule. There is no major reason to obey "the owner-computes" rule. The true objective is to distribute the parallel computations $S_{1}(i, j)$ and $S_{2}(i, j)$ to processor $P_{j}$, for $1 \leq i \leq n$. To this purpose, we might distribute columns of $a$ and $b$ to processors, which corresponds to Li and Chen's alignment. But we would insist that $S_{2}(i, j)$ is executed by processor $P_{j}$, at the price of a communication after the computation, to store the written value $b(j, i)$ into the memory of processor $P_{i}$. For each value of $i$, statement $S_{2}$ would then induce a gather operation ( $P_{j}$ owns $a(j, j)$, writes into $b(j, i)$ and sends it to $\left.P_{i}\right)$.

Remark 2: computations versus communications. Example 1 is a toy example and should be considered as such. In this example, our solution may not be significantly better than a solution that sequentializes the parallel loop, because of the cost of the communications. Still, we can easily modify the example! Also, we can take benefit of the many papers in the literature to derive the best physical distribution, i.e. deciding whether rows of $a$ and columns of $b$ will be distributed in a pure cyclic, pure block or block-cyclic fashion over $p$ physical processors, where $p$ is likely to be much smaller than $n$, the array size. In fact, our approach is quite flexible and can benefit from any precise modeling of the computation and communication costs: our alignment-distribution graph is vertex-weighted and edge-weighted, and the more precise the weights, the more accurate the solution. See the literature survey in Section 3.

Remark 3: loop parallelization algorithms and redistribution. An experienced programmer may have decided to apply loop distribution $[19, \text { p. } 323]^{1}$ on Example 1 before considering alignment and distribution. Such a transformation is perfectly legal and leads to the following loop nest:

\footnotetext{
${ }^{1}$ A confusing terminology! Loop distribution here amounts to distribute statements inside the same loop so that they appear in separate loops. It is not related to distributing loop instances to processors.
} 


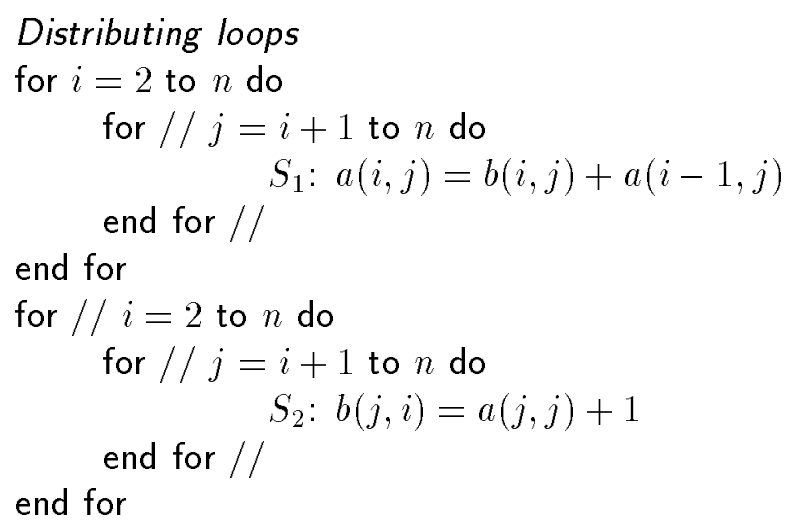

We could then perform the alignment step separately on the two nests, and eventually redistribute some data array ( $\operatorname{say} b$ ) in between. If the modified loop nest (having distributed the loop) is given as input to our alignment-distribution graph, and if the redistribution of one array (say $b$ ) is optimal, our algorithm will find it. But given the original loop nest of Example 1, we do not deal with ANY loop transformation.

Consider the following modification of Example 1:

\section{Example 2}

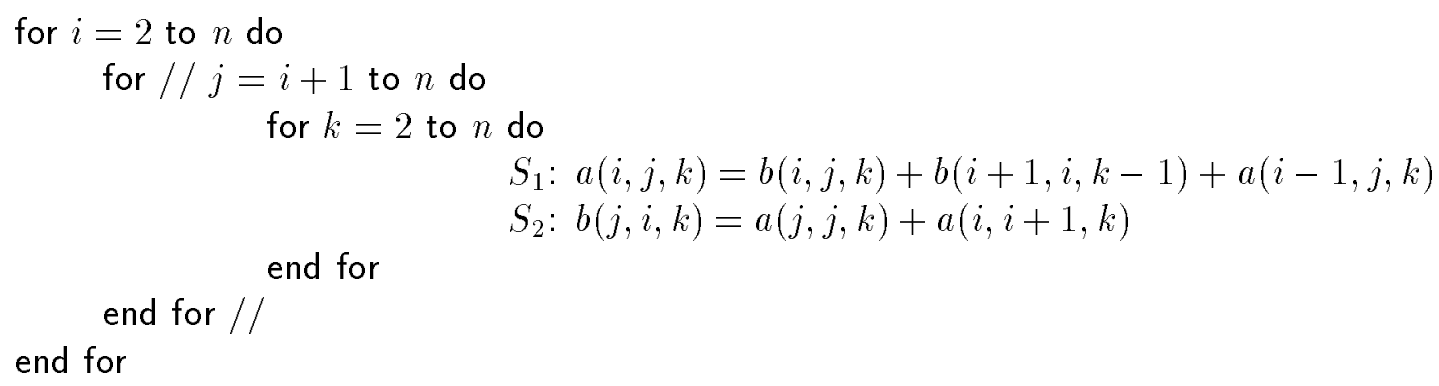

The reduced dependence graph is shown in Figure 4: loop distribution is no longer valid. We represent Li and Chen's CAG in Figure 5: solid arrows correspond to statement $S_{1}$, and dashed arrows to $S_{2}$. Again, the optimal solution for the CAG is to superimpose arrays $a$ and $b$, i.e. align each dimension of $a$ with the same dimension of $b$. Again, this would lead to a sequential execution, whatever the distribution chosen. However, as before, our alignmentdistribution graph, represented in Figure 6. gives priority to the parallel loop $j$ and distribute the first dimension of $a$ and the second dimension of $b$ to processors.

To summarize, our approach starts from a "parallelized" loop nest, i.e.a loop nest for which dependence analysis and loop parallelization have already been carried out. The most popular tools for these two steps are dependence levels [1, 2] and the Allen-Kennedy algorithm [2]. Given a parallelized loop nest, we determine which parallel loops should be distributed to processors, and the best alignment and distribution of arrays to minimize communications. This is done through the alignment-distribution graph.

Our main contribution is for a single loop nest, possibly non perfectly nested. When there are several consecutive loop nests, or an iterative loop surrounding several loop nests, we use the approach of Lee [11], which we briefly summarize in Section 4.3 when dealing with multiple nests. 


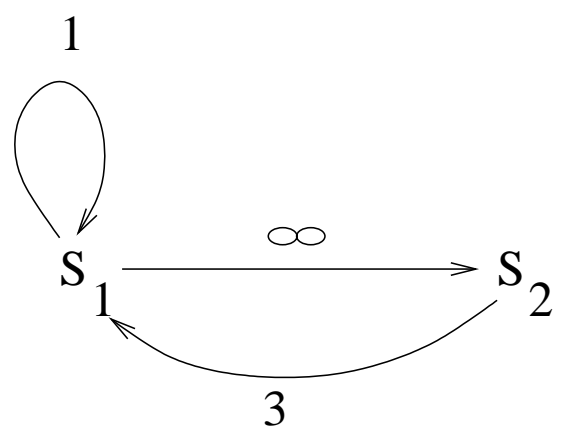

Figure 4: The reduced dependence graphs (using dependence levels) for Example 2.
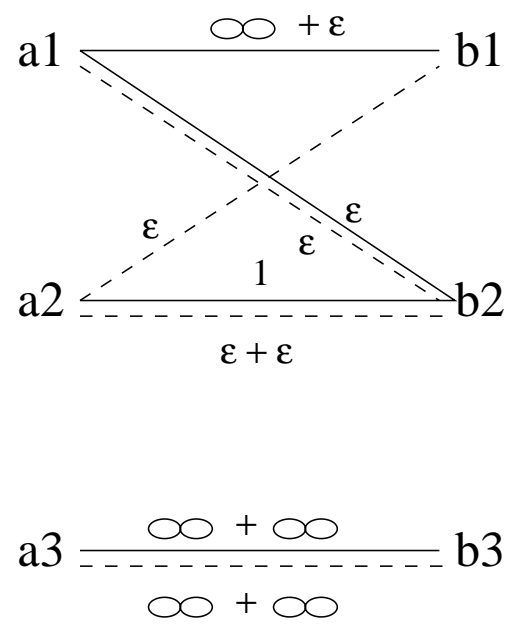

Figure 5: The component affinity graph for Example 2.

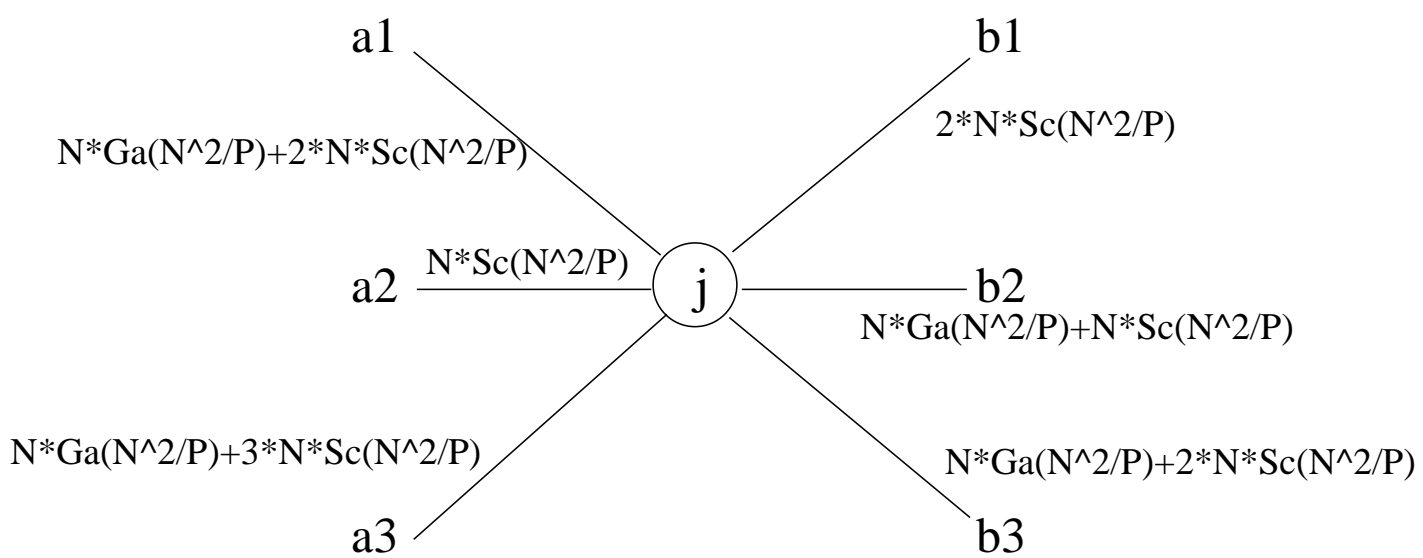

Figure 6: The alignment-distribution graph for Example 2. 


\section{Related work}

There are numerous papers on the alignment and distribution problem. We refer the reader to the survey [5] and the references therein. In this section, we summarize a few selected papers. In addition to Li and Chen's alignment method $[13,14]$ (already described in Section 2.1), we describe three papers by Tandri and Abdelrahman [17], Kelly and Pugh [9], and Ayguadé et al. [4, 3], whose goal is similar to ours. Next we present results by Gupta and Banerjee [7] and Li and Chen [12] on identifying structured communications and estimating their weight.

Our algorithm also uses the dynamic programming algorithm of Lee [11] when dealing with several loop nests. Indeed, redistributing some arrays between two consecutive nests may well prove more efficient. We describe Lee's technique in Section 4.3.

\subsection{Tandri and Abdelrahman}

Given a loop nest, Tandri and Abdelrahman [17] construct an undirected graph where each node represents either a parallel loop, or an array dimension. There is an edge between a loop node and an array node if the dimension considered is indexed by the loop variable.

Attributes are assigned to the nodes: ${ }^{*}$, Cyclic or CyclicRCyclic for loop nodes, to favor load balancing, and *, Block or BlockCyclic for array node, to favor local access. For example, if $X$ is referred to as $X(a * i+b * j)$ where $j$ (outer) is parallel and $i$ (inner) is sequential, then the attribute will be BlockCyclic.

There is a conflict when an edge connects two nodes whose attributes are different. To solve such a conflict, we replace the attributes by an intermediary. Thus, Cyclic and Block resolve to BlockCyclic.

Once all conflicts are solved, we have to assign dimensions of the processor geometry to the nodes. The algorithm is a greedy one. We consider first the outer loop. We assign to them and to the array nodes connected to them a dimension of processors. We pursue then with the other nodes. A distribution scheme is then found.

Tandri and Abdelrahman's method is somewhat crude, in that communication costs are not taken into account precisely. Also, their selection of the best array dimension to be distributed is not clear. Still, they give priority to distributing parallel lops, and next they align the array dimensions onto those loops: we believe this is the right way to go, and we use a similar (but refined) scheme in our algorithm.

\subsection{Ayguadé et al.}

Ayguadé et al. [4,3] consider programs constituted of several consecutive perfect loop nests $L_{1} L_{2} \cdots L_{n}$. All arrays are assumed to have the same dimension $d$. They describe their method for 1D- and 2D-grids, but we only deal with 1D-grids in this short survey. We start with the construction of a graph called the Communication-Parallelism Graph. Nodes are organized in columns. Each column represents an array in a nest and it contains $d$ nodes.

There are two types of edges. Data movement edges show possible alignment alternatives between the dimension of two arrays in a nest $L_{i}$. The assigned weight reflects the data movement cost to be paid if these two dimensions are aligned and distributed. We add other data movement edges to show possible realignment in a sequence of nests. If the array $A$ in $L_{i}$ is used in $L_{j}$, then $d \times d$ edges connect each node of array $A$ in $L_{i}$ to each node of $A$ in $L_{j}$. If the edge connects the same dimension, its weight is null, otherwise its weight is the cost of a realignment. 
Parallelism hyper-edges show possible parallelization strategies for the loops in $L_{i}$. An hyperedge connects the nodes corresponding to the array dimensions that have to be distributed to parallelize the loop according to the owner computes rule. Its weight is the time that is saved when the loop is parallelized.

We have to find a path in the CPG that includes exactly one node of each column so that the sum of weights of the edges minus the sum of weights of the hyper-edges that connect nodes in the chosen path is minimized. This problem is formulated as a linear $0-1$ programming problem. The variables are $Y_{P Q}(i, j)$ which corresponds to the edge between the $i^{\text {th }}$ dimension of $P$ and the $j^{t h}$ dimension of $Q$ and $Z_{k}$ which corresponds to the $k^{\text {th }}$ hyper-edge.

The constraints are the following:

- $\sum_{j} Y_{P Q}(i, j)=\sum_{j} Y_{Q R}(i, j) \forall i, P, Q, R$

- $\sum_{i} \sum_{j} Y_{P Q}(i, j)=1 \quad \forall P, Q$

- If $Z_{k}$ connects the nodes $X_{P^{1}}\left(i_{1}\right), \cdots, X_{P^{h}}\left(i_{h}\right)$ which are connected by the edges $Y_{P^{1} Q^{1}}, \cdots, Y_{P^{h} Q^{h}}$, we need $\sum_{j} Y_{P^{l} Q^{l}}\left(i_{l}, j\right) \geq Z_{k} \quad \forall l \in[1 . . h]$

The approach of Ayguadé et al. [4, 3] is interesting because of their precise estimation of edge weights. Also they can handle redistribution between consecutive nests. However, the requirement that all nests are perfect and that all arrays have same dimension is very restrictive. In addition, the integer linear programming solution may prove too expensive in practice.

\subsection{Kelly and Pugh}

The title of Kelly and Pugh's paper [9] is Minimizing communication while preserving parallelism. This title exactly corresponds to our goal! However Kelly and Pugh consider a framework quite different from ours: they study all the possible transformations (loop permutations) of the program to determine which one induces the maximum of parallelism and the best mapping of the computations.

To determine valid loop permutations, Kelly and Pugh use a dependence analysis more sophisticated than the dependence levels. The direct dependences are computed by the Omega software and the indirect dependences are computed by transitive closure.

For each legal permutation, they determine the parallelism level which is allowed and they estimate the number of required synchronizations (they use a sophisticated model which allows to take pipelining into account). Finally, for each statement pair, they compute the number of data written in the first statement and read in the second one, using value-based flow dependence analysis.

To summarize, in the case where a precise dependence analysis is possible (e.g. when all dependences are affine), Kelly and Pugh's method is quite powerful. However, it cannot be applied to general loop nests where only limited information (such as dependence levels) is available.

\subsection{Communication patterns}

Li and Chen [12] present interesting results on communication routines. They consider already parallelized programs with sequential and parallel loops. They assume that each array element 
can be assigned only once, that left-hand side subscripts are index variables, and that arrays are aligned to have a common index domain within each loop nest. We have a distribution scheme over a template and we want to recognize communication routines.

Each assignment $a\left(\sigma_{1}, \cdots, \sigma_{n}\right)=\cdots b\left(\delta_{1}, \cdots, \delta_{n}\right) \cdots$ may generate communications. If the tuples differ in only one corresponding pair of elements, the communication is either a Spread or a Reduce or a Copy or a Shift or a Multi-spread. The routine can be found with a pattern matching on these elements.

If the tuples are strongly different, we try by pattern matching on the tuples to recognize one of these routines: One-All-Broadcast, All-One-Reduce, Single-Send-Receive, Uniform-Shift or AffineTransform. When a pattern cannot be matched with a routine, we decompose it into sub-patterns. Indeed, a pattern over an n-dimensional index domain can be thought of as a composition of $n$ simple patterns. For example, send $a(c(i, j), j-3)$ to $(i, j)$ can be decomposed into two simple communications: send $a(c(i, j), j-3)$ to $(i, j-3)$, which is a Multi-spread, and then send (the data) from $(i, j-3)$ to $(i, j)$, which is a Shift.

Gupta and Banerjee [7] improve Li and Chen's alignment method to estimate communication costs. Their method is based on pattern-matching, applied upon the different assignments which could generate communications in the program. Their communication primitives are Transfer, OneToManyMulticast, ManyToManyMulticast, Scatter, Gather, Shift and Reduction.

They allow operations on the structure of the program to decrease the communications costs by founding a better placement of communication. For instance they use loop distribution over two components to enable any communication placed between those components to be aggregated with respect to that loop. They try to permute loops when there is a parallel loop outside a loop in which communication takes place. To control the size of communication buffers required, they propose to strip-mine the loops.

Sometimes, the compiler may generate more communication than necessary, for example when there are conditionals. Information about the frequency of execution of statements can help the compiler decide between carrying out potentially extra communication and using a large number of messages. Since the primitives corresponding to different terms implement the data movement in distinct grid dimensions, they can legally be composed in any order. So another optimization is to permute the communications in favor of reducing the message sizes handled by processors.

\section{Solving the alignment-distribution problem}

Al already stated, we start from a parallelized program, i.e. a program for which dependence analysis and loop parallelization have already been carried out: we are using the same hypotheses as Li and Chen [14]. Our goal is to preserve the potential parallelism while conducting the alignment step. We first describe our algorithm for a unidimensional processor grid. Next we move to a bidimensional grid. In both cases, we target a single (possibly non perfectly nested) loop nest. For several consecutive loop nests, we simply use the approach of Lee [11], who uses a dynamic-programming algorithm to determine whether some data redistribution is needed between two successive loop nests.

\subsection{Unidimensional grids}

\subsubsection{Construction of the alignment-communication graph}

We have two kinds of nodes in the graph, array dimension nodes and loop nodes: 
- For each array, each dimension of this array is represented by a node (like for Li \& Chen graph). The weight of such a node is zero.

- Each loop is also represented by a node. We give a weight to this node which represents the (approximated) execution time of the loop. For parallel loops, we divide the sequential execution time by the number of processors, as in Ayguadé et al [4, 3].

Edges link array dimension nodes to loop nodes. There is an edge between two such vertices if there is a reference to the corresponding array dimension in the corresponding loop; the edge weight represents the (estimated) communication costs induced by the distribution of both the array dimension and the loop instances to the processors.

Finally, we add dashed arrows to illustrate the loop nesting. This is only for convenience. We refer to loop nodes and dashed arrows as the loop subgraph of the alignment-communication graph.

Consider the Cholesky factorization algorithm showed in Example 3. We use this example to describe our algorithm because it is a classical in compilation literature. Data dependence analysis can be conducted exactly on this example because all references are affine, but this is by no means a requirement for our algorithm.

\section{Example 3}

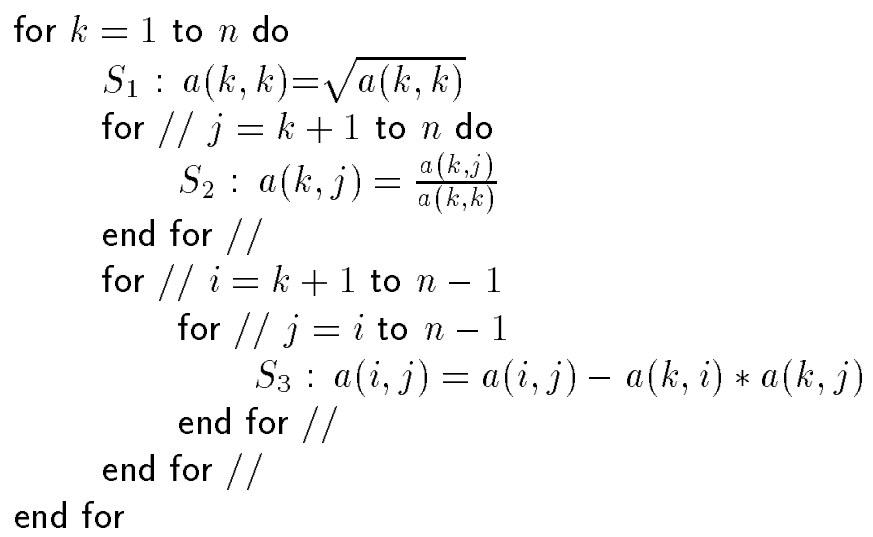

Note that Li and Chen's CAG for Cholesky has no edge, because there is a single array in the nest, and they do not take self references into account. We represent the alignment-distribution graph in Figure 7. Boxed nodes are the loop nodes: we use a circle for a parallel loop and a square for a sequential loop. The other nodes are the array dimension nodes. We use the following routines for the edge weights:

$$
\begin{aligned}
\operatorname{Br}(N)=\operatorname{Broadcast}(N) & \text { a processor sends the same } N \text { data items. } \\
\operatorname{Sc}(N)=\operatorname{Scatter}(N) & \text { a processor sends } N \text { different data items. } \\
\operatorname{Ga}(N)=\operatorname{Gather}(N) & \text { a processor receives } N \text { different data items. } \\
\text { Aap }(N)=\text { All_to_all_personalized }(N) & \text { each processor sends } N \text { different data items. } \\
\operatorname{Aa}(N)=\text { All_to_all }(N) & \text { each processor sends the same } N \text { data items. }
\end{aligned}
$$

For example, the edge between $a 2$ and the left parallel node $j$ comes from statement $S_{2}$. It means that if we distribute this $j$ loop and the second dimension of $a$, each processor $j$ which computes $a(k, j)$ has to receive from the same processor $k$ the value of $a(k, k)$; hence the label $\operatorname{Br}(1)$. 


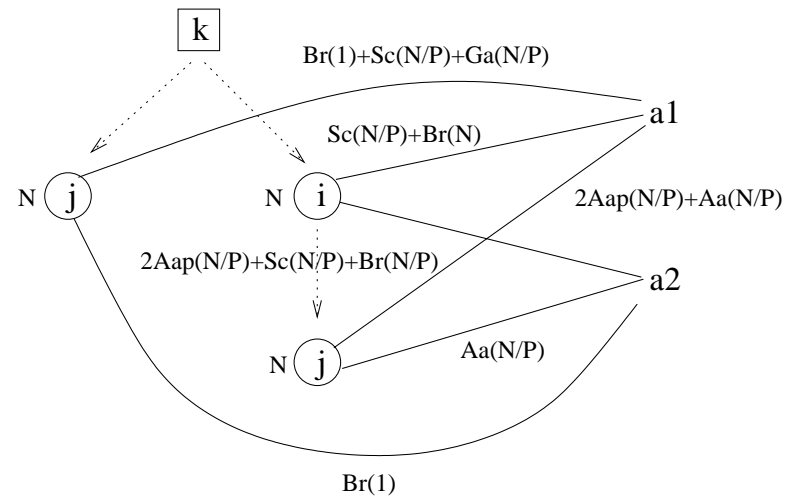

Figure 7: The alignment-distribution graph for Example 3.

\subsubsection{The algorithm}

The goal is to find exactly one parallel loop node to distribute, along each path of the loop subgraph. We also need to distribute a dimension of each array. The optimization criteria is to minimize residual communications costs.

The optimal solution is to consider all different possibilities to distribute the parallel loops. Once a given distribution is chosen, we compare for each array the communication costs generated by this distribution, and we select the dimension which minimizes the communications. We sum the costs over all arrays and we obtain the total cost of the selected loop distribution. We keep the loop distribution scheme of minimal cost.

Coming back to Example 3, there are two different paths. We have to choose $j$ in the left path, and either $i$ or $j$ in the right path. In the case of the distribution scheme $(j, i)$, we have for $a 1$ the weight $N * B r(1)+2 N * S c(N / P)+N * G a(N / P)+N * B r(N)$ and $N * B r(1)+$ $2 N * \operatorname{Aap}(N / P)+N * S c(N / P)+N * B r(N / P)$ for $a 2$. The weight of $a 1$ is lower, hence we distribute $a 1$. For the other distribution scheme $(j, j)$, the weight is $N * B r(1)+N * S c(N / P)+$ $N * G a(N / P)+2 N * A a p(N / P)+N * A a(N / P)$ for $a 1$ and $N * B r(1)+N * a a(N / P)$ for $a 2$. In this case, we choose $a 2$. Then we have to compare the two solutions. The cost of the first solution is $N * B r(1)+2 N * S c(N / P)+N * G a(N / P)+N * B r(N)$, and the cost of the second solution is $N * B r(1)+N * a a(N / P)$. Since a personalized all-to-all is expensive, we would most certainly select the first solution.

\subsubsection{Complexity}

Consider first the case of a perfect loop nest. Let $s$ be the number of parallel loops, $T$ be the number of arrays and $d_{i}$ the dimension of the $i$-th array $T_{i}$. The complexity of our algorithm is $\mathcal{O}\left(s \times \sum_{i=1}^{T} d_{i}\right)$ because for each parallel loop and for each array, we search for the best dimension to distribute. Letting $d=\max _{i}\left(d_{i}\right)$ be the largest array dimension, the complexity of our algorithm is $\mathcal{O}(d \times T \times s)$.

It is important to understand why this result does not contradict the NP-completeness result of Li and Chen, who show that the alignment problem is NP-complete in the size of the CAG, i.e. the number of arrays $T$ multiplied by the largest array dimension $d$. The intuitive explanation is the following: $\mathrm{Li}$ and Chen have no template reference for the alignment problem, so they have to explore the possibility of aligning each dimension of each array with every dimension of every other array, hence the combinatorial swell. On the contrary in our approach, because we aim at preserving 
the potential parallelism, each loop distribution scheme constitutes a reference pattern for which we search the best distribution for each array. Because we have few possible loop distribution schemes, the overall complexity is kept small.

Theorem 1 The alignment-distribution problem can be solved in time $\mathcal{O}(d \times T \times s)$ for a perfect loop nest with $s$ parallel loops and $T$ arrays with largest dimension $d$.

In the case of a non-perfect nest, on a given path labeled $i$ in the loop nodes of the alignmentdistribution graph, there are $s_{i}$ parallel loops. For instance in Example /refprog.choles, we have two paths in the loop subgraph, $s_{1}=1$ and $s_{2}=2$. The complexity of the algorithm is $\mathcal{O}\left(d \times T \times \prod_{i=1}^{p} s_{i}\right)$ because $\prod_{i=1}^{p} s_{i}$ represents the number of distribution scheme. In the worst case, the complexity is $\mathcal{O}\left(d \times T \times e^{s}\right)$.

The exponential term is not important. Indeed, the number of parallel loops in a nest is not higher than 3 in practice.

\subsubsection{Remarks}

Remark 1 In the above version of the algorithm, we always distribute exactly one parallel loop along each path of the loop subgraph. In certain cases, it may well be more efficient to execute a parallel loop in sequential mode on a single processor. We can implement this modification, which amounts to select at most one (instead of exactly one parallel loop along each path of the loop subgraph: we make a copy of each parallel node. One copy indicates a sequential execution and the other a parallel execution. So, there are twice as many loop nodes, hence more loop distribution schemes to evaluate.

Similarly, we always distribute one dimension of each array. Sometimes, it will be better to allocate a whole array to an unique processor. To that purpose, we can add a node for each array which indicates that we do not want to distribute this array.

Remark 2 The problem (and of course the alignment-communication graph) is "symmetric" between loop nodes and array dimension nodes. Sometimes, it will be better to iterate on all possible distribution schemes for the arrays, and to deduce the best distribution scheme for the loops. For Example 3, there is a single array of dimension 2 and several loop nodes, so we should indeed consider the different choices for distributing $a$, and for each of them to determine the best distribution scheme for the loops.

Remark 3 For the (mostly theoretical) situation where our algorithm would be too costly, we can introduce the following greedy heuristic: along each path of the loop subgraph, give priority to distributing the most external parallel loop. This will lead to the largest granularity of the tasks that are distributed to processors.

\subsection{Bidimensional grids}

If the dimension of the processor grid of processors is larger than one, we propose the following two strategies. 


\subsubsection{Recursive algorithm}

We build the alignment-distribution graph just as in Section 4.1, and we use the previous unidimensional algorithm. At this stage we have chosen to distribute one parallel loop and one dimension of each array. We distribute them along the first dimension of the grid.

We construct a new graph by deleting already chosen nodes. We update edge weights by taking the distribution scheme for the first grid dimension into account. Then we use a second time the unidimensional algorithm to determine which loops and which array dimensions will be distributed along the second grid dimension.

We iterate the process as many times as there are dimensions in the processor grid.

\section{Example 4}

Assume that we target a $2 \mathrm{D}$-processor grid for the following nest:

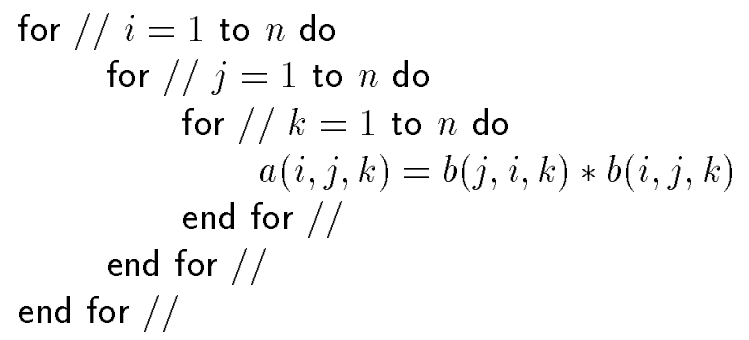

Using this recursive algorithm, we first distribute the $k$ loop and the last dimension of $a$ and $b$. Indeed, such a choice preserves the parallelism and is communication-free. After deleting the corresponding nodes and updating the weights, we obtain the graph of Figure8. Next the recursive algorithm decides to distribute $i$ and the first dimension of $a$ and $b$ along the second grid dimension.

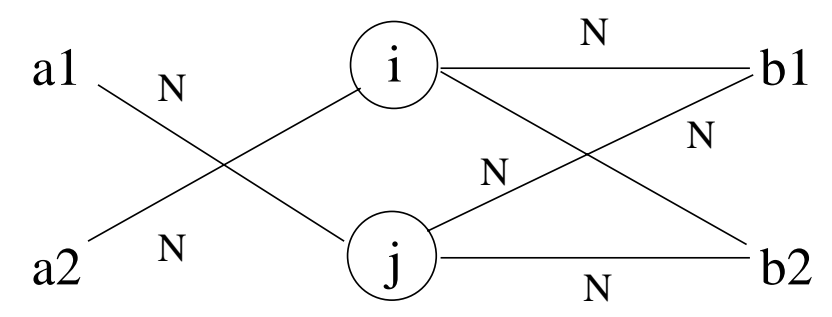

Figure 8: Recursive algorithm : after the first step.

\subsubsection{Optimal algorithm}

The main principle of the optimal algorithm is the same as in the unidimensional case. Instead of considering one node by path of the loop subgraph, we consider $g$ nodes by path, where $g$ is the dimension of the target processor grid. When $d$ loop nodes are chosen along each path, we determine for each dimension of each array the cost of the communications induced by the distribution of this dimension and these loops. We keep the loop distribution scheme which minimizes the communications. 


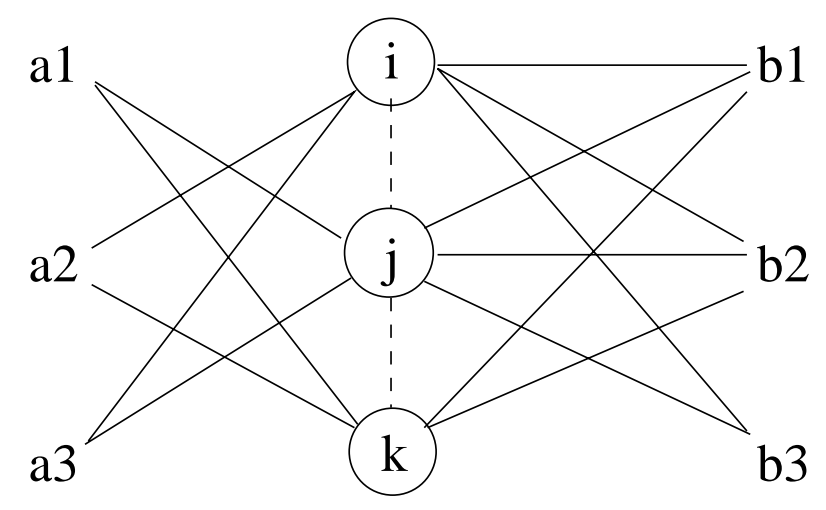

Figure 9: The alignment-distribution graph for Example 4.

Coming back to the example 4, we construct the graph depicted in the figure 9 . In this graph, we have to compare the three following cases: distribute $(i, j)$, distribute $(i, k)$ or distribute $(j, k)$.

Distribute $(i, j):$ We distribute $a 1, a 2$ and $b 1, b 2$.

Distribute $(i, k):$ We distribute $a 1, a 3$ and $b 1, b 3$.

Distribute $(j, k):$ We distribute $a 2, a 3$ and $b 2, b 3$.

In all three cases communications come from accessing $b(j, i, k)$. The first case is very expensive. We have to choose between the second and the third. Since the communications are the same for both, we distribute $(i, k)$, the solution with largest task granularity.

\subsubsection{Comparison}

Let $g$ be the number of dimensions of the processor grid. For the recursive algorithm, the complexity for a perfect loop nest is $\mathcal{O}(g \times d \times T \times s)$. For a non perfect nest, we get $\mathcal{O}\left(g \times d \times T \times e^{\frac{s}{e}}\right)$. This is because we use the unidimensional algorithm $g$ times. Of course $g$ can be viewed as a small constant in practice ( $g=2$ or 3 for current machines).

For the optimal algorithm, the complexity for a perfect nest is $\mathcal{O}(\#$ schemes $\times T \times d)$. The number of loop distribution schemes is $\left(\begin{array}{c}g \\ s\end{array}\right)$. Hence the complexity is $\mathcal{O}\left(d \times T \times s^{g}\right)$. For a non perfect nest, the complexity is $\mathcal{O}\left(d \times T \times \prod_{i=1}^{p} s_{i}^{g}\right)$. So in the worst case, it's $\mathcal{O}\left(d \times T \times e^{g \times s}\right)$.

Of course the optimal algorithm has higher complexity. However, it relies on a more accurate estimation of the communication costs, because when we search for a loop distribution scheme we look for $g$ dimensions of arrays to distribute together with the selected loops.

\subsection{Several nests}

In the case of several loop nests, we use the method proposed Lee [11]. Given a program constituted by a sequence of $n$ nests, we want to determine the best distribution scheme (for parallel loops and arrays) for the whole program. In a word, Lee [11] uses Li and Chen's CAG as a basic block for a single loop nest, together with a dynamic programming algorithm to determine whether to 
redistribute some array in between two consecutive blocks. We simply suggest to use our alignmentdistribution graph as a new basic block, and to keep the dynamic approach unchanged. This will preserve parallelism over the whole program in addition to determining the best distribution and re-distribution of arrays.

When we consider two consecutive nests, we have two main choices:

- either we keep the same alignment-distribution for the two nests, and we look for the scheme that minimizes the sum of the communications for both nests,

- or we determine the best alignment-distribution for each nest, and we use a redistribution in between.

Consider a sequence of $n$ loop nests $L_{1} L_{2} \cdots L_{n}$. For each subsequence $L_{i} L_{i+1} \cdots L_{i+j-1}$, where $1 \leq i \leq n, 1 \leq j \leq n-i+1$. Let $T_{i, j}$ be the minimal time to compute $L_{1} L_{2} \cdots L_{i+j-1}$ with the restriction that it uses the distribution scheme $P_{i, j}$ for the sequence $L_{i} L_{i+1} \cdots L_{i+j-1}$. Thus the final distribution scheme after computing $T_{i, j}$ is $P_{i, j}$. At the beginning, $T_{1, j}$ is equal to $M_{1, j}$. Let $\operatorname{cost}\left(P_{i-k, k}, P_{i, j}\right)$ be the communication cost of changing data layouts from $P_{i-k, k}$ to $P_{i, j}$. Lee [11] uses the following dynamic programming algorithm:

$$
\begin{aligned}
& \text { for } i=2 \text { to } s \text { do } \\
& \quad \text { for } j=1 \text { to } s-i+1 \text { do } \\
& \qquad T_{i, j}=\min _{1 \leq k<i}\left(T_{i-k, k}+M_{i, j}+\operatorname{cost}\left(P_{i-k, k}, P_{i, j}\right)\right) \\
& \quad \text { end for } \\
& \text { end for } \\
& \text { Minimum }=\min _{1 \leq k \leq s}\left(T_{s-k+1, k}\right)
\end{aligned}
$$

If the sequence of nests is enclosed by an iterative loop, the last line of the algorithm is modified as follows:

$$
\text { Minimum }=\min _{1 \leq k \leq s}\left(T_{s-k+1, k}+M A X_{-} I T E R \times \text { dependence }\left(T_{s-k+1, k}\right)\right),
$$

where dependence $\left(T_{s-k+1}\right)$ returns the cost of changing data layouts from the distribution scheme of the last nest to the first one.

Consider the following simple example :

\section{Example 5}

$$
\begin{aligned}
& \text { for } / / i=1 \text { to } n \text { do } \\
& \text { for } j=1 \text { to } n \text { do } \\
& a(i, j)=a(i, j-1)+a(i, j) \\
& \text { end for } \\
& \text { end for } / / \\
& \text { for } i=1 \text { to } n \text { do } \\
& \text { for } / / j=1 \text { to } n \text { do } \\
& a(i, j)=a(i-1, j) * a(i, j) \\
& \text { end for } / / \\
& \text { end for }
\end{aligned}
$$

Lee's algorithm consists in considering the program either as a unique nest or as two nests for which we may need to determine a redistribution scheme. 
A unique nest: Our alignment-distribution algorithm decides to distribute the two parallel loops and the first dimension of $a$. The second nest induces many communications.

Two different nests: For the first nest, we distribute the $i$ loop and the first dimension of $a$. For the second nest we distribute the $j$ loop and the second dimension of $a$. There is no communication inside the two nests, but we need communications to redistribute $a$ between them.

We have to compare both solutions. In the first case, processor $P_{j}$ receives $a(i, j)$ from $P_{i}$ and $a(i-1, j)$ from $P_{i-1}$, and then sends the result to $P_{i}$. Each processor has to communicate with all the others several times. However, if we use a block distribution, these communications are often transformed into local memory accesses. So the final solution is to distribute $i, j$ et $a 1$ (the unique nest strategy).

\section{Conclusion}

We have introduced the alignment-distribution graph to replace Li and Chen's component affinity graph. The major two advantages of our approach are the following:

- Parallelism is preserved: we derive the best loop distribution together with the best array alignment

- Complexity is polynomial for perfect loop nests. Complexity is always polynomial in the number of arrays addressed inside the nest.

In addition, we retain all the flexibility of Li and Chen's approach: new results from the literature and from experiments can be easily incorporated, for instance to refine the estimation of the communication and computation weights. Indeed, our weight model for communications is much more refined than the original $\mathrm{CAG}$ of $\mathrm{Li}$ and Chen; as for computation costs, we can also benefit from the literature, e.g. $[8,15,16]$. Finally, our graph can be used as a building block for techniques that manipulate larger programs.

The current largest limitation is that our alignment-distribution graph is built for a fixed, already parallelized loop nest. It would be nice to incorporate loop transformations in the framework: how to determine the best way of writing the loop nest, in order to derive the best way to distribute arrays and computations to processors?

\section{References}

[1] John R. Allen and Ken Kennedy. PFC: a program to convert Fortran to parallel form. Technical Report MASC-TR82-6, Rice University, Houston, TX, USA, 1982.

[2] John R. Allen and Ken Kennedy. Automatic translation of Fortran programs to vector form. ACM Transactions on Programming Languages and Systems, 9(4):491-542, October 1987.

[3] E. Ayguadé, J. Garcia, M. Gironès, M. L. Grande, and J. Labarta. DDT: A research tool for automatic data distribution in HPF. Scientific Programming, 6(1):73-94, 1997.

[4] E. Ayguadé, J. Garcia, M. Gironésa, J. Labarta, J. Torres, and M. Valero. Detecting and using affinity in an automatic data distribution tool. In Languages and Compilers for Parallel Computing, pages 61-75. Springer-Verlag, 1995. 
[5] E. Ayguadé, J. Garcia, and U. Kremer. Tools and techniques for automatic data layout: a case study. Parallel Computing, 24:557-578, 1998.

[6] Michael R. Garey and Davis S. Johnson. Computers and Intractability, a Guide to the Theory of NP-Completeness. W. H. Freeman and Company, 1991.

[7] M. Gupta and P. Banerjee. Demonstration of automatic data partitioning techniques for parallelizing compilers on multicomputers. IEEE Transactions on Parallel and Distributed Systems, 3(2):179-193, 1992.

[8] Mohammad R. Haghighat. Symbolic Analysis for Parallelizing Compilers. Kluwer Academic Publishers, 1995.

[9] W. Kelly and W. Pugh. Minimizing communication while preserving parallelism. In Proceedings of the 10th ACM International Conference on Supercomputing. ACM Press, 1996.

[10] Charles H. Koelbel, David B. Loveman, Robert S. Schreiber, Guy L. Steele Jr., and Mary E. Zosel. The High Performance Fortran Handbook. The MIT Press, 1994.

[11] PeiZong Lee. Efficient algorithms for data distribution on distributed memory parallel computers. IEEE Transactions on Parallel and Distributed Systems, 8(8):825-839, 1997.

[12] J. Li and M. Chen. Compiling communication-efficient programs for massively parallel machines. IEEE Transactions on Parallel and Distributed Systems, 2(3):361-375, 1991.

[13] Jingke Li and Marina Chen. Index domain alignment: Minimizing cost of cross-referencing between distributed arrays. In Frontiers 90: The 3rd Symposium on the Frontiers of Massively Parallel Computation, pages 424-433, College Park, MD, October 1990. IEEE Computer Society Press.

[14] Jingke Li and Marina Chen. The data alignment phase in compiling programs for distributedmemory machines. Journal of Parallel and Distributed Computing, 13:213-221, 1991.

[15] Kathryn S. McKinley. Automatic and Interactive Parallelization. PhD thesis, Department of Computer Science, Rice University, 1992.

[16] W. Pugh. Counting solutions to Pressburger formulas: how and why. In ACM SIGPLANConference on Programming Language, Design and Implementation. ACM Press, 1994.

[17] S. Tandri and T. S. Abdelrahman. Automatic data and computation partitioning on scalable shared memory multiprocessors. In 3rd Workshop on Automatic Data Layout and Performance Prediction, 1997.

[18] Michael Wolfe. The Tiny loop restructuring research tool. In H.D. Schwetman, editor, International Conference on Parallel Processing, volume II, pages 46-53. CRC Press, 1991.

[19] Michael Wolfe. High Performance Compilers For Parallel Computing. Addison-Wesley, 1996. 